\title{
Au sujet d'une observation de Colladon au pont de la Machine à Genève
}

\author{
On an observation by Colladon \\ at the machine bridge Geneva
}

\author{
par G. DARRIEUS, \\ MENHIR DE H'INSTITUT.

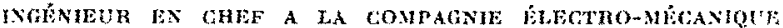

\begin{abstract}
Interprétation physique (*) d'un tourbillon hélico-centripète à axe horizontal observé sus. le Rhone a tienève immédiatement en amont d'un barrage et pour certaines dispositions d'onnerlare des rideanx.

Cette circulation al sein d'un cicoulement presque irrotationnel trouverait son origine dans la préexistence d'une rotation due au frottement sur le lond du lit et telle que la révele le profil des vitesses. Le phenomine hydranlique pent être assimilé a certains phénomènes atmosphériques, tels que les trombes et les colonnes de fumée de grand diamètre, dont l'auteur troube une explication dans la rotation terrestre, génératrice des cyclones tropicaux. Enfin, un autre phénomène hydraulique du méme genve est celui de la prise d'eau froide de l'energie ther. mique des mers décrit ici méme il y a quelques annees pur les Ets Neyrpic ${ }^{+\cdots}$
\end{abstract}

Bien que le ponl de la Machine existe toujours, il ne semble pas que les Gènevois aient gardé Io souvenir du «serpent d'eau » décrit par CoLLA-

(*) Linterpretation mathèmatique du phènomène fait l'objet d'un mémoire antérieur de M. G. Danneus : * Entretien par extension suivant son axe, d'une trombe rectiligne dans un huide visqueux. $\gg$ (Extrait des Mémoires sur la Mécanique des Fluides, offerts it M. D. Riabovchnsky, à l'occasion de son jubilé scientifique. Publications scientifiques et techniques du Ministère de lair « hors série 》)

Un eertain nombre de ces extraits obligeamment offerts par l'auteur au Comité teehnique de la S.H.F. sont déposés au secrétariat ef peuvent être adressés aux membres intéressés, sur demande et jusquu'à épuisement.

(*) A. Craya et P. Ganiel: \& Recherches theoriques et expérimentales sur l'éconlement dans un fluide hétirogène stratifé. $\gg$ (Mémoire présenté au Comité technique de la S.H.F. le 19 novembre 1946 et public dans to compte rendu du Congrès de la Hécanique Appliquée de 1946.)

\begin{abstract}
Physical interpretation (*) of a heluo-centri. petal, horizontal vortex observed in the Rhone at Geneva immediately unstream from a dam and for cerlain dispositions of the gate opening. The circulation in the midalle af an irrotational flow would originate from the diflusion of turbulence date to friction on the bottom as is reveated by the velocity distribution. The hydratie phenomenon might be compared to certain almo. spheric phenoment, such as balerspouts ant the torge diameter columns of smoke, for mhich the anthor finds an explomation in the earth's rotation, generator of tropical 'yclone's. Finally another hydratic phenomenon of the some tyme is that of the cold ander intake thermal enera!n from the sea, described in la Houille Blanehe by Ets Negrpic (") some llears ago.
\end{abstract}

now en 1890 (1), peut-être parce qu'il ne se produit que pour certaines dispositions des ouvertures du barrage a rideaux.

Rappelons que ce phénomène consiste en l'apparition, à environ un mètre au front du barrage, d'un long tourbillon horizontal de 8 it $15 \mathrm{~m}$, que rend visible le noyau d'air très approximativement eylindrique, de 1 à $10 \mathrm{~cm}$ de diamètre, déterminé par la cavitation. Bien que le phénomène commence aux extrémités, en deux troncons qui se rejoignent d'ailleurs en un temps tris court, la forme de ces extrémités, recourbées vers l'aval, ne joue évidemment pas de rôle essentiel, sinon pour rendre compte pent-itre des condi-

(1) Daniel Cothadox. - Sur whe tromise d'eau dont la bouche est en bas, dont le corps est horizontal et peut avoir quinze cents fois son diametre. Arch. d. Sc. Phys. et Nat, t. XXIV, août 1890 , Genève, et $C, k$. Ac. d. Sc. t. III, p. 449 (1890); egatement mentionnd dans le comrs de Physique de Botasse: Tourbillons, 1, p.95. 
tions d'un accès éventuel plus ou moins étranglé de lair extérieur au noyau de la trombe, dans lequel règne une dépression en général modérée, qui peut suffire à expliquer un entretien du noyau d'air par dégagement de l'air dissous.

Si Colladon, dans sa controverse avec l'astronome FayE, insiste sur ce que ces extrémités, sans doute par suite de l'ouverture partielle des rideaux, sont courbées vers le bas, c'est pour contester la généralité, affirmée par FAYE, de l'orientation opposée observée dans d'autres cas, par exemple dans les entonnoirs qui débouchent it la surface des eaux courantes.

Mais la circonstance essentielle dont dépend l'explication du phénomène, réside bien entendu dans les conditions amont, à savoir l'existence, au sein du courant fluide, d'une circulation autour du noyau, laquelle, en l'absence d'aucun organe tel que volute ou aube directrice (") susceptible de créer cetle circulation dans un écoulement irrotationnel, ne peut trouver son origine que dans une rotation distribuée au sein du lluide, telle qu'en détermine normalement, par diffusion de la turbulence, le frottement sur le fond du lit, et telle que la révèle le profil des ritesses.

Dans ces conditions, le mouvement hélico-centripète, quasi de révolution au voisinage du noyau, que détermine l'étirement des filets de courant et de tourbillon de part et d'autre du plan de symétrie, doit donner lieu, suivant le théorème d'Hexunoltz, en l'absence de dissipation, à une augmentation continue au cours du temps de l'intensité tourbillonnaire et des vitesses de circulation; d'où résulte l'apparition d'une cavitation.

Notre calcul qui traite pour simplifier le cas, valable au roisinage du noyau, d'un écoulement strictement de révolution, s'appliquerait plus exactement à l'espace aval des directrices d'une turbine centripète Francis ou Kaplan, en labsence de la roue, ou, dans le cas d'un rotationnel préexistant au sein du fluide, à la vidange, par un trou central, d'un réservoir cylindricue lournant autour d'un axe vertical.

Le résultat de ce calcul est la mise en évidence de ec que, comme il b́tait à attendre, les vitesses en jeu ne sont pas si faibles que la viscosité de lean ait une influence sensible sur l'évolution de l'ecoulement, sauf au voisinage immédiat de laxe du tourbillon, ou de la surface du noyau quand il $y$ a cavitation; de sorte que l'explication du phénomène demeure indépendante, dans ses grandes lignes, du caractire non parfait du

( ) Pent-tre toutefois le decollement de la couche limite que détermine sur le fond, au voisinage de lobstacle, le ralentissement du coumant fig. 1 de notre mémoire precité jone-t-il ce rôle dans une certaine mesure. fluide, si toutefois l'existence préalable d'une rotation qui, elle, résulte de la viscosité et de la turbulence, est considérée comme donnce.

Toutefois la persistance évenluelle, an voisinage du noyau, malgré les faibles vitesses en jeu et le caractère à peu près laminaire de l'écoulement, d'une certaine turbulence résiduelle superposée à la rotation d'ensemble, pourrait amener à substituer à la viscosité vraie une viscosité apparente beaucoup plus grande, au sens de Reynolds, Boussinfso, Prandte, G. I. Taylon, etc.

Bovasse consacre plusieurs pages de son grand Traité de Physique (Tourbillons, t. V) à des observations de trombes atmosphériques. ColcaDoN raconte ainsi avoir vu en juillet, vers midi, les menus objets d'une lessive abondante étendue sur le sol d'une place, subitement enlevés en tourbillonnant jusqu'à une hauteur d'environ $600 \mathrm{~m}$, et disperses au loin par une trombe qui paraissait avoir au plus $2 \mathrm{~m}$ de diamètre. Picter déerit également en détail les trombes de 2 a $3 \mathrm{~m}$ de diamètre et paraissant atteindre 500 à $1000 \mathrm{~m}$ de hauteur qu'il a observées en Egypte au voisinage du Caire, de mars à octobre, au milieu du jour, et qui, rendues visibles par le sable entraîné, paraissent alimentées par la couche d'air surchauffé qui surmonte le sol, où un thermomètre enfoui marque $84^{\circ}$, tandis que la température de l'air, $35^{\circ}$ à l'ombre, atteint $52^{\circ}$ au centre de la trombe.

REDField (1839) décril, parmi trois cas d'immenses feux de branchages, celui concernant un amas circulaire bien sec provenant, après enlèvement du bois de charpente, du défrichement de 14 hectares de forêt, et allumé sur tout le pourtour, un jour d'été parfaitement calme. La colonne de fumée, s'élevant verticalement aussi haut que la vue pouvail attcindre, et accompagnée d'un crépitement sourd, coupé d'explosions comme le roulement du tonnerre, tourbillonnait avec une vitesse suffisante pour soulever à une vingtaine de mètres de jeunes arbres de $20 \mathrm{~cm}$ de diamètre couchés sur le sol.

Des observations analogues ont été faites lors de grands incendies de bâtiments en bois, ou de forêts, notamment dans les Landes. Malheureusement, aucune des relations ci-dessus ne comporte l'indication du sens de rotation.

Or, bien que l'on puisse douter à première vue, avec Bouasse, que la rotation terrestre, cause manifeste des grands cyclones tropicaux, doive expliquer aussi les phénomènes à plus petite échelle ci-dessus, on voit mal quelle autre cause fortuite mais soutenue pendant des heures (de 10 h. du matin à 3 h 30 de l'après-midi, dans le eas d'une des trombes de sable décrites par PıcTET), lelle qu'une rotation locale préexistante ou dissumétrie du vent, pourrait rendre compte. par 
lemps calme, de la régularité et de la persistance de ces phénomènes.

La composante zénithale $\omega \sin : 0^{\circ}$ de la rotation terrestre a la latitude 50", environ 5,5.10-1 $\mathrm{rad} / \mathrm{s}$, correspond, pour un rayon de $100 \mathrm{~m}$ dams le plan horizontal, a une vitesse ar d'entrainement du sol par rapport au système de référence d'orientation fixe, de $5,5 \cdot 10^{-4} \cdot 10^{2}=5,5 \mathrm{~cm} / \mathrm{s}$. II suffit de supposer que les masses d'air primitivement au repos relatif sur un cercle de ce rayon, se trouvent aspirées par la trombe sur un rayon cent fois plus petit, pour rendre compte, par simple conservation de la circulation, de l'apparition de vitesses tangenticlles de $5,5 \mathrm{~m} / \mathrm{s}$ au contour même de la trombe de un mètre de rayon. L'hypothèse d'un rayon initial de $1000 \mathrm{~m}$, soit dix fois plus grand, conduirait à des vitesses finales cent fois plus grandes.

Il est viai que l'appel en moins d'une heure de masses d'air aussi éloignées que même $100 \mathrm{~m}$, peut paraître invraisemblable, si le foyer aspirant, feu ou trombe, se comporte comme un "puits $\%$ au sens aterodynamique, c’est-à-dire détermine, comme on le vérifie d'ailleurs autour d'une crépine, un champ de vitesses induites à symétrie sphérique. Le demi-volume d'une sphère de $100 \mathrm{~m}$ de rayon, 2 millions de $\mathrm{m}^{3}$, est en effel bien supérieur à celui qui peut être aspiré par la trombe dans le temps de sa formation.

Mais la difficulté est levée s'il est admis que l'aspiration s'exerce seulement sur la couche d'air plus chaude et plus légère située au voisinage du sol, et dont le volume, pour un rayon de $100 \mathrm{~m}$ et une épaisscur d'un mètre, n'est que de $7800 \mathrm{~m}^{3}$, de sorle que le débit de $6 \mathrm{~m}^{3} / \mathrm{s}$ (dû par exemple à un courant ascendant de $2 \mathrm{~m} / \mathrm{s}$ dans une trombe de $3 \mathrm{~m}^{2}$ de section) suffirait à faire apparaitre en vingt minutes la rotation calculée plus haut de $5,5 \mathrm{~m} / \mathrm{s}$.

Une telle aspiration sclective peut résulter de l'instabilité naturelle de l'atmosphère, qui se développe par l'effel de l'échaufement solaire, lorsque le gradient vertical de température a dépassé le gradient adiabatique, normalement rẻalisé pour l'air d'humidité moyenne dans la troposphère. L'appel d'air constitué par la trombe rasscmble alors en un seul la multitude des filets chauds qui, demeurant dispersés en son absence, se frayent un chemin vers le haut à travers les masses plus froides, pour tendre à rétablir le gradient adiabatique.

The autre raison de cet appel d'air par tranches horizontales on cylindriques, qui fait décroitre la composante centripète de la vitesse te long du rayon $r$ en $1 / r$, c'est-à-dire beaucoup plus lentement que lappel spherique en $1 / r^{2}$ d'un "puits», est l'effet d'èjecteur étudié par PRANDTL. et calculé par Tondures, associé à l'épanouissement d'un jet tel que le réalise la colonne de feu ou la trombe.

L'aspiration n'est alors plus localisée an foyer ou au pied de la trombe, mais plus ou moins distribuée sur toute la hauteur de la colonne ascendante.

Enfin, un troisiome cas de conditions \& baroclines $\gg$ où la pression n'est pas fonction uniforme de la densité, et qui échappe à l'application génerale du théorème de CauchY-HeLnholT\%, est celui, analogue à la prise d'eau froide de l'́nergie thermique des mers étudié el décrit ici-même il y a quelques années par la Société Neyrpic, ou une inversion du gradient vertical de temperalure au voisinage du sol, plus froid que l'air, par l'effet du rayonnement nocturne, ríalise, comme dans la stratosphère, une stabilité naturelle, dans le champ de la pesanteur, des couches inférieures stratifices, que tend à respecter l'appel du foyer el de la trombe.

C'est ainsi que l'expetience a rofuté les objections, dailleurs assez peu fondées en principe. de ceux qui doutaient, à l'origine du procédé Crat'de-Botchenot, de la possibilité d'aspirer l'eau froide des profondeurs, sans provoquer ì la longue l'appel simultané des eaux plus chaudes de surface.

Dans le cas du serpent d'eau, un des résultats de notre calcul est de montrer qu'en écoulement laminaire, le freinage de la circulation auquel donne lieu au voisinage du noyau la viscosite, avec reflux par diflusion, de l'intensité tourbillonnaire rers l'amont, ne suffit pas a consommer l'apport convergent de celte inlensité Lourbillonnaire dans le cals où elle próexiste au sein du fluide, ni ì expliquer que celte circulation n'augmenle pas au cours du lemps commo dans le cas des trombes aturosphériques dues a la rotation de la lerre.

Il parait nécessaire d'admetlre que lo caratetère sensiblement irrotationnel de l'écoulement est dû à ce que les filets liquides qui concourent à alimenter la trombe, proviennent en majeure partie de la tranche superieure rapide du courant amont, celle qui contient le sommet cou maximum) du profil des vitesses ef au voisinage duquel le gradient vorlical de la vitesso en lequel se réduit ici le rotationnel, est fable, voire négatif (près de la surface); landis que les flets tourbillonnaires mais plus lents, voisins du fond, ne contribueraient que pen ou point a celte alimentation. 


\section{DIS CUSSION}

M. le President Hupnen remereie M. Danmeus ot le felicite d'avoir fait un savant exposé sans en exclure une facile intelligibilite. II indique que, d'accord avec M. Barnillon, il a prié M. Delattre de se renseigner it Genève sur la persistance du phénomène constaté par Colladon vers $1890\left({ }^{*}\right)$.

M. le Président se demande s'il n'y a pas eu d'observations sur les fumées provoquées par l'incendie des dépôts de pétrole dans la région de Rouen en 1940 et si l'on peut rapprocher le phénomène des observations faites par l'Administration des Eaux et Forèts dans les Landes où la défense contre le teu consiste parfois à allumer un autre feu.

M. Callet rappelle que, selon certains témoignages, les trombes dues à la stratifieation des couches d'air au cour des incendies monstres de Hambourg entrainaient it renversaient les hommes.

M. de Rovville pense qu'une indication sur la manifestation de trombes produites par le feu pourrait être donnee par l'inclinaison des câbles des ballons-sondes captifs. Il remarque que le site de Genève, placé dans une cuvette de montagnes, doit etre favorable à la formation de ces trombes. D'autre part, la répartition aes vitesses dans l'écoulement liquide considéré influe sans doute sur la formation du tourbillon, bien qu'il soit difficile de réaliser un écoulement de vitesse constante sur toute la profondeur.

M. Bergeron confirme que, dans un courant, l'inégalité des vitesses peut créer un mouvement de rotation dont l'axe est perpendiculaire à l'écoulement; par exemple, pour une pompe à axe vertical, le decalage de la tubulure d'aspiration par rapport à l'axe du canal damenée crée dans ec dernier un déséquilibre des ritesses qui engendre dans certains cas un mouvement de rotation genre Vortex dans la tuyauterie d'aspiration de lit

(*) Les renseignements, atressés postérieurement à la séance, par M. DelattRe à M. le President Hernek, confment la persistance du phénomène dans certains cas douperture des vames. pompe. Ce mouvement de rotation contrarie l'entréc normale de l'enu dans la roue et peut rendre la pompe bruyante.

Sur la demande de M. Bengenon, M. Darreus rappelle que l'existence d'une circulation dans les turbo-machines est due a la formation de tourbillons fictifs, non portés par le fluide mais liés a la forme de l'aube, de sorte que le fluide se retrouve, à la sortie de la couronne distributrice, avec un mouvement dont la génératrice est, dans le cas d'un écoulement plan, une spirale logarithmique, mouvement irrotationnel, mais qui a une cireulation.

M. Je Président se demande si le rapport de la profondeur it la larseur de l'obstacle joue un rôle. Il suggère ì M. Darnieus de demander à M. Blosset et à M. Grlaent, ingenieurs en chef des Services de la Navigation de la Seine a Paris, si des phenomènes de ce genre ont pu etre observes sur les barrages mobiles en service sur la Seine.

M. Rementeras signale qu'un serpent d'eau existait à la prise d'eau de la Glaire près de Luchon. Il essatera d'obtenir des renseignements à ce sujet si, toutefois, la prise n'a pas été modifiée entre temps.

M. Le Président suggère de demauder à M. Escaxbr, qui a fait des études sur modèles réduits de barrages à plusieurs passes, s'il n'y a pas observé un phénomène semblable à celui décrit par M. Darrueus et qui d'après celui-ci, pourrait être épuré et rendu plus accessible à In théorie dans une étude sur modèle.

M. Rextenenas rapproche l'observation de Coldador de celle relatée par il. Swovin dans son ouvrage : « La préparation et l'épandage simultanes des liants routiers $\gg(* *)$. Dans ses expériences sur un type de eyclone dit « cylindre-spiral », M. SmoNis a constaté la formation dans l'axe dudit cylindre, d'un Vortex aéré analogue au « serpent d'eau $\geqslant$ etudie par M. Darrievs.

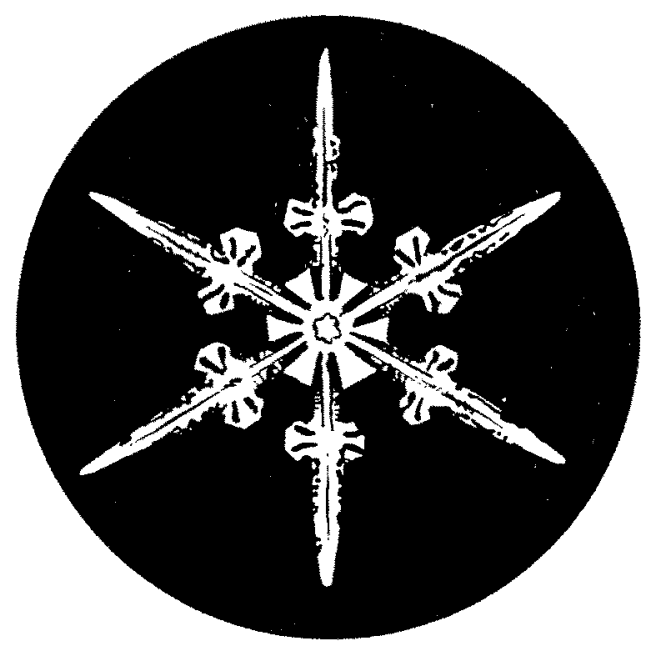

